Gut and Liver, Vol. 11, No. 2, March 2017, pp. 298-305

\title{
Efficacy of Capecitabine Plus Oxaliplatin Combination Chemotherapy for Advanced Pancreatic Cancer after Failure of First-Line Gemcitabine-Based Therapy
}

\author{
Kwang Hyun Chung, Ji Kon Ryu, Jun Hyuk Son, Jae Woo Lee, Dong Kee Jang, Sang Hyub Lee, and Yong-Tae Kim \\ Department of Internal Medicine and Liver Research Institute, Seoul National University College of Medicine, Seoul, Korea
}

Background/Aims: Second-line chemotherapy in patients with advanced pancreatic ductal adenocarcinoma (PDAC) that progresses following gemcitabine-based treatment has not been established. This study aimed to investigate the efficacy and safety of second-line combination chemotherapy with capecitabine and oxaliplatin (XELOX) in these patients. Methods: Between August 2011 and May 2014, all patients who received at least one cycle of XELOX (capecitabine, 1,000 $\mathrm{mg} / \mathrm{m}^{2}$ twice daily for 14 days; oxaliplatin, $130 \mathrm{mg} / \mathrm{m}^{2}$ on day 1 of a 3-week cycle) combination chemotherapy for unresectable or recurrent PDAC were retrospectively recruited. The response was evaluated every 9 weeks, and the tumor response rate, progression-free survival and overall survival, and adverse events were assessed. Results: Sixty-two patients were included; seven patients (11.3\%) had a partial tumor response, and 20 patients (32.3\%) had stable disease. The median progression-free and overall survival were 88 days (range, 35.1 to 140.9 days) and 158 days (range, 118.1 to 197.9 days), respectively. Patients who remained stable longer with frontline therapy ( $\geq 120$ days) exhibited significantly longer progression-free and overall survival. The most common grade 3 to 4 adverse events in patients were vomiting (8.1\%) and anorexia (6.5\%). There was one treatmentrelated mortality caused by severe neutropenia and typhlitis. Conclusions: Second-line XELOX combination chemotherapy demonstrated an acceptable response and survival rate in patients with advanced PDAC who had failed gemcitabinebased chemotherapy. (Gut Liver 2017;11:298-305)

Key Words: Carcinoma, pancreatic ductal; Treatment outcome; Capecitabine; Oxaliplatin; Salvage therapy

\section{INTRODUCTION}

Pancreatic ductal adenocarcinoma (PDAC) is well known as an aggressive malignancy, and is mostly diagnosed at advanced stage, which makes it unsuitable for curative resection. ${ }^{1-3}$ Even after a curative resection, recurrence rates are high and median overall survival (OS) of patients reaches only 15 to 19 months. ${ }^{4}$ For unresectable or recurrent PDAC, anticancer chemotherapy is the mainstay of treatment and gemcitabine is the key agent. Several gemcitabine-based regimens are established as standard chemotherapy; ${ }^{5}$ however, the efficacy of those regimen is unsatisfactory. ${ }^{6,7}$ FOLFIRINOX regimen which is a combination of oxaliplatin, 5-fluorouracil (5-FU) and irinotecan demonstrated a better outcome than a gemcitabine-based regimen; however, because of the substantial increase in toxicity, only selected patients who had good performance status could be therapeutic targets. ${ }^{8}$ Various alternative gemcitabine-based regimens, which added cytotoxic chemotherapeutic agents such as cisplatin and capecitabine or targeted agents such as sorafenib and bevacizumab, were tried. However, these attempts, too, failed to show satisfactory results. ${ }^{9-12}$ A gemcitabine-based regimen in combination with albumin-bound paclitaxel improved patient survival. However, median progression-free survival (PFS) of patients was only 5.5 months. ${ }^{13}$

High failure rate and short duration of disease control of firstline treatment regimens in PDAC patients prompted the need for second-line treatment regimens. Current guidelines only vaguely recommend to switch to fluoropyrimidine-based regimens when treatment with gemcitabine-based first-line regimens fails. ${ }^{14}$ There have been several studies on second-line regimens for patients whose cancers progress after treatment with gemcitabinebased regimens. Several phase II studies investigated the effi-

Correspondence to: Ji Kon Ryu

Department of Internal Medicine and Liver Research Institute, Seoul National University Hospital, Seoul National University College of Medicine, 101 Daehak-ro, Jongno-gu, Seoul 03080, Korea

Tel: +82-2-2072-1962, Fax: +82-2-762-9662, E-mail: jkryu@snu.ac.kr

Received on June 20, 2016. Revised on July 25, 2016. Accepted on July 25, 2016. Published online December 16, 2016

pISSN 1976-2283 eISSN 2005-1212 https://doi.org/10.5009/gnl16307

@) This is an Open Access article distributed under the terms of the Creative Commons Attribution Non-Commercial License (http://creativecommons.org/licenses/by-nc/4.0) which permits unrestricted non-commercial use, distribution, and reproduction in any medium, provided the original work is properly cited. 
cacy of 5-FU and oxaliplatin combination therapy, and reported a response rate of up to 23\%, a PFS of 6 to 22 weeks, OS of 15 to 31 weeks. ${ }^{15-17}$ More recently, a phase III study using 5-FU, leucovorin, and oxaliplatin for a 6-week cycle showed better outcomes than use of 5-FU and leucovorin without oxaliplatin (PFS, 2.9 months vs 2.0 months; OS, 5.9 months vs 3.3 months, respectively). ${ }^{18}$ On the other hand, there has been a recent study of therapy using nanoliposomal irinotecan in combination with 5-FU and leucovorin, that showed longer survival than therapy with 5-FU and leucovorin alone (6.1 months vs 4.2 months). ${ }^{19}$

Chemically, capecitabine is oral fluoropyrimidine carbamate designed to generate 5-FU preferentially in tumor tissue, through exploitation of high intratumoral concentrations of thymidine phosphorylase. ${ }^{20}$ It has proved to be active for therapy in PDAC. ${ }^{21}$ Thus, a combination of capecitabine and oxaliplatin (XELOX) is also expected to be as effective as the combination of 5-FU and oxaliplatin, used as a second-line regimen for PDAC patients whose cancers have progressed after treatment with a gemcitabine-based regimen. XELOX combination chemotherapy has advantages over oral chemotherapy with a combination of 5-FU and oxaliplatin. However, very few phase II studies exist $\mathrm{t}^{22,23}$ and more data are needed. Therefore, we aimed to evaluate the efficacy and adverse effects of XELOX combination chemotherapy in pancreatic cancer patients whose cancer had advanced or recurred, despite initial gemcitabinebased therapy.

\section{MATERIALS AND METHODS}

\section{Patients}

We consecutively included all patients who received XELOX combination chemotherapy as salvage treatment for advanced or recurred pancreatic cancer, between August 2011 and May 2014, at our institution (Seoul National University Hospital, tertiary referral hospital). All included patients had cancer that had progressed after a gemcitabine-based chemotherapy. All of the patients had Eastern Cooperative Oncology Group (ECOG) performance status of 0 to $2,{ }^{24}$ and had adequate hepatic, renal, and bone marrow function.

Demographic data, initial cancer stage, initial chemotherapeutic regimen, performance status at initiation of XELOX combination chemotherapy, and prior treatment modalities were identified by retrospective review of medical records. Duration of initial chemotherapy was also reviewed and 120 days were used as a cutoff to discriminate patients who were stable for long or short periods with frontline therapy which was adopted from previous study. ${ }^{25}$ The seventh edition of the tumornode-metastasis system from the American Joint Committee on Cancer was used to determine the clinical stage of the study patients. $^{26}$

\section{Chemotherapy}

XELOX combination chemotherapy consisted of oxaliplatin, (dosage, $130 \mathrm{mg} / \mathrm{m}^{2}$ ) given intravenously as a 120-minute infusion on day 1 , and capecitabine (dosage, $1,000 \mathrm{mg} / \mathrm{m}^{2}$ ), given orally, two times daily for 14 days. Chemotherapy was administered at the outpatient clinic and the patients were followed up every 3 weeks; this was defined as a cycle of therapy. Treatment response after chemotherapy was determined by the revised Response Evaluation Criteria in Solid Tumors (RECIST) guidelines (version 1.1). ${ }^{27}$ Tumor response was evaluated at baseline, and at every 9 weeks thereafter, by pancreatic protocol computed tomography. In patients who had significant comorbidities or poor performance (ECOG performance status 2), dosage was as follows: oxaliplatin, $110 \mathrm{mg} / \mathrm{m}^{2}$; and capecitabine, $750 \mathrm{mg} /$ $\mathrm{m}^{2}$. XELOX combination chemotherapy was continued until the tumor progression occurred, or the patient had unacceptable toxicity, or refused further chemotherapy.

Dose modifications and treatment delays were based on observed drug-related toxicity, and the attending physician's assessment. Adverse events were assessed according to the Common Terminology Criteria for Adverse Events, version 3.0. ${ }^{28}$ The study protocol was based on the Declaration of Helsinki and was approved by the Institutional Review Board of Seoul National University Hospital (IRB approval number: H-1407-109596).

\section{Statistical analyses}

Data are shown as the number (in percentages) for categorical variables, the mean \pm standard deviation for continuous variables, and as median (95\% confidence interval) for PFS and OS of patients. The Kaplan-Meier method and log-rank test were used to compare the PFS and OS. To identify the independent factors associated with these outcomes, a Cox proportional hazard model was used for the multivariate analysis. All of the survival analyses were performed as an intention-to-treat analysis; thus, all of the enrolled patients were included in the analysis. Two-tailed p-values of $<0.05$ were considered to be statistically significant. All analyses were performed using SPSS version 21.0 software (IBM Corp., Armonk, NY, USA).

\section{RESULTS}

\section{Patient characteristics}

During the study period, a total of 62 patients were administered XELOX combination chemotherapy as salvage treatment for advanced pancreatic cancer that had progressed after initial gemcitabine-based chemotherapy. The baseline characteristics of these patients are described in Table 1 . The mean age of patients was $61.4 \pm 9.7$ years; of this, 36 (58.1\%) were male and 26 (41.9\%) were female. Initial cancer stage was stage III (locally advanced disease) in 27 patients (43.5\%) and stage IV (metastatic 
disease) in 25 patients (40.3\%). In 10 patients (16.1\%), tumor had recurred after curative resection, and one patient received palliative resection for residual tumor. Initial chemotherapy was gemcitabine and erlotinib regimen in 43 patients (69.4\%), gemcitabine single regimen in 16 (25.8\%), and gemcitabine plus cisplatin regimen in three (4.8\%). Concurrent chemoradiotherapy was performed in 15 of the 62 patients (24.2\%). Tumor status at the time of failure of first-line gemcitabine-based treatment (or, conversely, at the start of XELOX combination therapy) was locally advanced tumor in 32 patients (51.6\%), and metastatic disease in 30 of them (48.4\%). ECOG performance status score was 0 in six patients (9.7\%), 1 in 30 (48.4\%), and 2 in 26 (41.9\%).

Table 1. Baseline Characteristics of Study Patients

\begin{tabular}{|c|c|}
\hline Characteristic & XELOX $(n=62)$ \\
\hline \multicolumn{2}{|l|}{ Sex } \\
\hline Male & $36(58.1)$ \\
\hline Female & $26(41.9)$ \\
\hline Age, yr & $61.4 \pm 9.7$ \\
\hline \multicolumn{2}{|l|}{ Initial cancer stage } \\
\hline III & $27(43.5)$ \\
\hline IV & $25(40.3)$ \\
\hline Recurred tumor & $10(16.1)$ \\
\hline \multicolumn{2}{|l|}{ Initial chemotherapeutic regimen } \\
\hline Gemcitabine+erlotinib & $43(69.4)$ \\
\hline Gemcitabine monotherapy & $16(25.8)$ \\
\hline Gemcitabine+cisplatin & $3(4.8)$ \\
\hline Surgical resection & $11(17.7)$ \\
\hline Concurrent chemoradiotherapy & $15(24.2)$ \\
\hline \multicolumn{2}{|c|}{ Tumor status at failure of first-line treatment } \\
\hline Locally advanced & $32(51.6)$ \\
\hline Metastatic & $30(48.4)$ \\
\hline \multicolumn{2}{|l|}{ ECOG } \\
\hline 0 & $6(9.7)$ \\
\hline 1 & $30(48.4)$ \\
\hline 2 & $26(41.9)$ \\
\hline \multicolumn{2}{|l|}{ Comorbidity } \\
\hline Hypertension & $15(24.2)$ \\
\hline Diabetes mellitus & $29(46.8)$ \\
\hline Chronic hepatitis B & $2(3.2)$ \\
\hline Atrial fibrillation & $1(1.6)$ \\
\hline COPD & $1(1.6)$ \\
\hline Parkinsonism & $1(1.6)$ \\
\hline Asthma & $1(1.6)$ \\
\hline Cerebrovascular disease & $3(4.8)$ \\
\hline Dx. to secondary chemotherapy, day & $241(56-913)$ \\
\hline
\end{tabular}

Data are presented as number $(\%)$ or mean \pm SD or median (range). ECOG, Eastern Cooperative Oncology Group; COPD, chronic obstructive pulmonary disease; Dx., diagnosis.
Median number of days between initial diagnosis and initiation of secondary chemotherapy (i.e., XELOX) was 241 (range, 56 to 913).

\section{Treatment response and adverse events}

At least one cycle of XELOX combination chemotherapy was administered to every patient, and a median of three cycles (range, 1 to 21 cycles) were delivered to patients. Initial tumor response to XELOX combination chemotherapy could be evaluated in 49 patients (79.0\%) after 9 weeks (three cycles) of treatment initiation. Treatment response by RECIST criteria was partial response in seven patients (11.3\%), stable disease, in 20 (32.3\%), and progressive disease, in 22 (35.5\%). Response could not be evaluated in 13 patients (21.0\%). Among them, nine stopped further chemotherapy before completion of the three cycles of XELOX chemotherapy, due to onset of adverse events (five patients experienced vomiting; one duodenal ulcer bleeding) or deterioration of general medical condition (in three patients). Four patients were lost to follow-up after the first cycle of chemotherapy. Tumor control rate (partial response or stable

Table 2. Tumor Response to Chemotherapy

\begin{tabular}{lc}
\hline & XELOX $(\mathrm{n}=62)$ \\
\hline $\begin{array}{l}\text { Treatment response (after 3 cycles) } \\
\text { Partial response }\end{array}$ & $7(11.3)$ \\
Stable disease & $20(32.3)$ \\
Progressive disease & $22(35.5)$ \\
NA & $13(21.0)$ \\
Tumor control rate & $27(43.5)$ \\
Delivered cycles of chemotherapy & $3(1-21)$ \\
\hline
\end{tabular}

Data are presented as number (\%) or median (range).

NA, not available.

Table 3. Nonhematologic Adverse Events after the First Three Cycles of XELOX

\begin{tabular}{lccc}
\hline & Grade 1 & Grade 2 & Grade 3 \\
\hline Anorexia & 4 & 6 & 4 \\
Vomiting & 2 & 4 & 5 \\
Diarrhea & 2 & 3 & 2 \\
Oral mucositis & 1 & 0 & 1 \\
Fatigue & 1 & 3 & 2 \\
Paresthesia & 7 & 2 & 1 \\
Insomnia & 1 & 1 & 0 \\
Nausea & 1 & 1 & 0 \\
Palmar-plantar & 0 & 2 & 0 \\
$\quad$ erythrodysesthesia syndrome & & & \\
Abdominal pain & 1 & 3 & 0 \\
Myalgia & 0 & 1 & 0 \\
Constipation & 2 & 0 & 0 \\
\hline
\end{tabular}


disease) was 43.5\% with intention-to-treatment analysis, and 55.1\% with per protocol analysis (Table 2). Adverse events after first three cycles of XELOX combination chemotherapy are described in Table 3. The most common grade 3 to 4 adverse event was vomiting (in $8.1 \%$ patients) followed by anorexia (in 6.5\% patients). There was one treatment related mortality, caused by severe neutropenia and typhlitis.

\section{PFS and OS after initiation of XELOX combination chemotherapy}

Median PFS after initiation of XELOX combination chemotherapy was 88 days (range, 35.1 to 140.9 days) (Fig. 1). Patients who remained stable for longer with frontline therapy (more than 120 days) had significantly longer PFS; this is the only significant factor which correlated with longer PFS in both univariate analysis (PFS, 61 days [range, 53.9 to 68.1 days] in $<120$ days vs 116 days [range, 53.0 to 179.0 days] in $\geq 120$ days, $\mathrm{p}=0.021$ ), and multivariate analysis (hazard ratio [HR] of 0.297 [range, 0.116 to 0.761 ] in $\geq 120$ days). Younger age, better tumor status at failure of first-line treatment, and initial gemcitabine combined with erlotinib chemotherapy regimen failed to yield statistically significant result in multivariate analysis (Table 4).

Median OS after initiation of XELOX combination chemotherapy was 158 days (range, 118.1 to 197.9 days) (Fig. 2). Factors that significantly correlated with longer survival in univariate analysis were better tumor stage at diagnosis or failure of first-line treatment, and longer duration between diagnosis and secondary chemotherapy ( $\geq 120$ days). In multivariate analysis, significant factors related with longer survival were younger

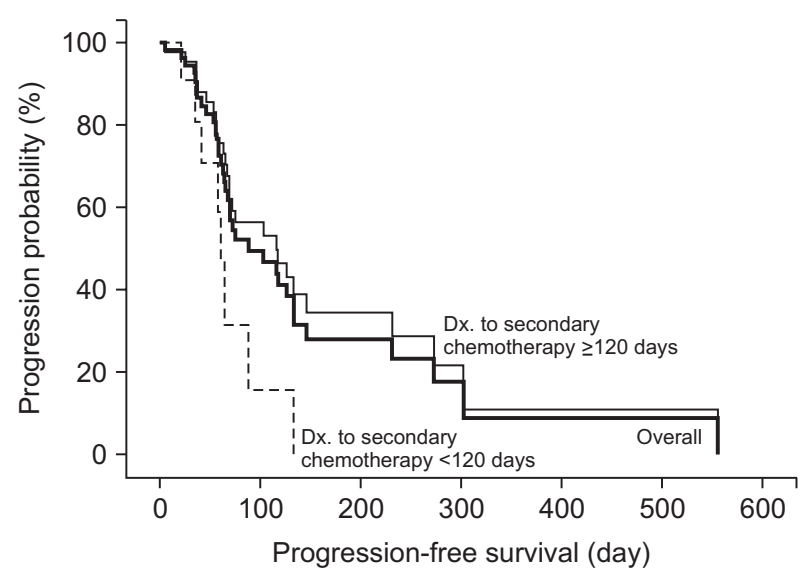

Fig. 1. Kaplan-Meier estimation of progression-free survival. The median progression-free survival of patients was 88 days (range, 35.1 to 140.9 days). Patients who remained stable longer with frontline therapy (>120 days) had significantly longer progression-free survival (61 days [range, 53.9 to 68.1 days] in $<120$ days vs 116 days [range, 53.0 to 179.0 days] in $\geq 120$ days, $p=0.021$ ). Solid line indicates patients who remained stable for more than 120 days, and broken line indicates patients who remained stable less than 120 days. Thick curve indicates overall patients.

Dx., diagnosis. age, better tumor stage at failure of first-line treatment, gemcitabine combined with erlotinib as first-line chemotherapy, and longer duration between diagnosis and secondary chemotherapy ( $\geq 120$ days) (Table 5).

\section{DISCUSSION}

Second-line chemotherapy for PDAC is yet to be elucidated. Most recent guidelines recommend fluoropyrimidine-based therapy for patients initially treated with gemcitabine-based therapy. ${ }^{14}$ Several fluoropyrimidine-based combination regimens were tried, and a recent study demonstrated that use of the fluoropyrimidine, 5-FU, in combination with oxaliplatin for therapy resulted in better survival than use of 5-FU alone. ${ }^{18}$ We expected that capecitabine can be used instead of 5-FU in the fluoropyrimidine and oxaliplatin combination regimen. In this study, we demonstrated a tumor control rate of $43.5 \%$, a PFS of 88 days (range, 35.1 to 140.9 days), and a median OS of 158 days (range, 118.1 to 197.9 days), when we used XELOX as a secondary chemotherapy regimen in patients with advanced or recurrent PDAC whose cancer had progressed with initial gemcitabine-based therapy.

Earlier phase II trials using XELOX as second-line therapy for PDAC showed a tumor control rate of 28.6\%, a PFS of 9.9 weeks (range 9.6 to 14.5 weeks) and median OS of 23 weeks (range, 17.0 to 31.0 weeks) with 41 patients. ${ }^{22}$ These results are inferior to ours with respect to tumor control rate, and comparable, in case of PFS and OS. The difference in study population may be the cause of this. We included a number of ECOG PS 2

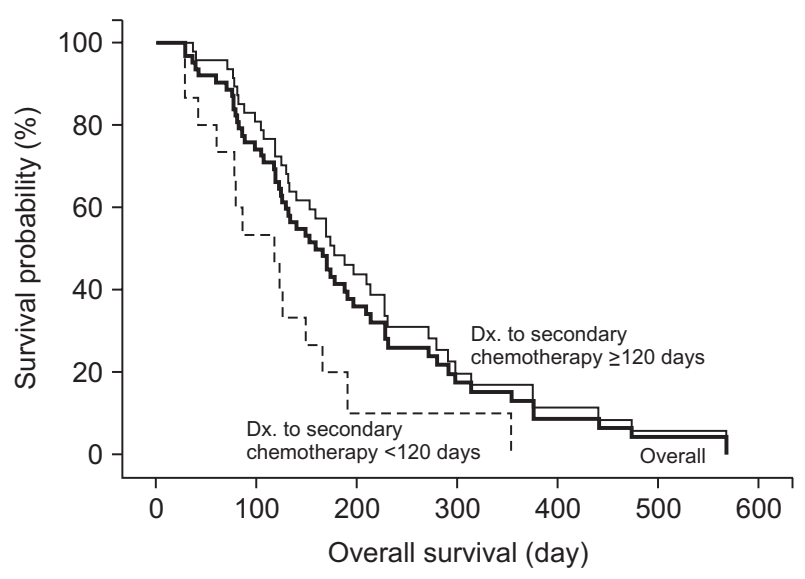

Fig. 2. Kaplan-Meier estimation of overall survival. The median overall survival of patients was 158 days (range, 118.1 to 197.9 days). Patients who remained stable longer at frontline therapy (more than 120 days) had significantly longer overall survival (117 days [range, 62.7 to 171.3 days] in $<120$ days vs 177 days [range, 136.5 to 217.5 days] in $\geq 120$ days, $p=0.006$ ). Solid line indicates patients who remained stable more than 120 days, and broken line indicates patients who remained stable less than 120 days. Thick curve indicates overall patients. 
Table 4. Factors Related to Progression-Free Survival

\begin{tabular}{|c|c|c|c|c|}
\hline & \multicolumn{2}{|c|}{ Univariate analysis } & \multicolumn{2}{|c|}{ Multivariate analysis } \\
\hline & Median PFS (95\% CI) & p-value & HR (95\% CI) & $\mathrm{p}$-value \\
\hline Sex & & 0.141 & & 0.259 \\
\hline Male & $126(51.8-200.2)$ & & 1 & \\
\hline Female & $72(50.6-93.4)$ & & $1.694(0.678-4.230)$ & \\
\hline Age, yr & & 0.386 & & 0.276 \\
\hline$<65$ & $116(57.1-174.9)$ & & 1 & \\
\hline$\geq 65$ & $72(45.3-98.7)$ & & $1.490(0.727-3.050)$ & \\
\hline Initial tumor stage & & 0.261 & & \\
\hline Recurred tumor & $56(30.7-81.3)$ & & & \\
\hline Stage III & $116(47.5-184.5)$ & & & \\
\hline Stage IV & $103(32.8-173.2)$ & & & \\
\hline Tumor status at failure of first-line treatment & & 0.184 & & 0.097 \\
\hline Locally advanced & $116(44.0-188.0)$ & & 1 & \\
\hline Metastatic & $69(11.2-126.8)$ & & $1.970(0.884-4.391)$ & \\
\hline ECOG performance status & & 0.762 & & \\
\hline 0 & $67(0-268.6)$ & & & \\
\hline 1 & $103(41.8-164.2)$ & & & \\
\hline 2 & 75 (58.2-91.8) & & & \\
\hline Hypertension & & 0.964 & & \\
\hline No & $75(38.5-111.5)$ & & & \\
\hline Yes & $116(55.2-176.8)$ & & & \\
\hline Diabetes & & 0.503 & & \\
\hline No & $116(45.8-186.2)$ & & & \\
\hline Yes & $69(22.6-115.4)$ & & & \\
\hline Initial chemothrapeutic regimen & & 0.052 & & 0.076 \\
\hline Gemcitabine+erlotinib & $133(50.9-215.1)$ & & 1 & \\
\hline Gemcitabine monotherapy & $67(50.9-83.1)$ & & $2.879(1.153-7.193)$ & \\
\hline Gemcitabine+cisplatin & $88(6.4-169.6)$ & & $1.236(0.288-5.301)$ & \\
\hline Diagnosis to secondary chemotherapy, day & & 0.021 & & 0.012 \\
\hline$<120$ & $61(53.9-68.1)$ & & 1 & \\
\hline$\geq 120$ & $116(53.0-179.0)$ & & $0.297(0.116-0.761)$ & \\
\hline Surgical resection & & 0.356 & & \\
\hline No & $103(50.5-155.5)$ & & & \\
\hline Yes & $56(30.9-81.1)$ & & & \\
\hline Concurrent chemoradiotherapy & & 0.858 & & \\
\hline No & $116(51.1-180.9)$ & & & \\
\hline Yes & $72(52.1-91.9)$ & & & \\
\hline
\end{tabular}

PFS, progression-free survival; CI, confidence interval; HR, hazard ratio; ECOG, Eastern Cooperative Oncology Group.

patients, in contrast to the former, which included only a small number of ECOG PS 2 patients (41.9\% vs 28.6\%). Further, we included only $48.4 \%$ of metastatic patients but the earlier study included almost exclusively metastatic patients (95\%). A recent study has demonstrated an overall disease control rate of 38.3\%, PFS of 12 weeks (range, 9.8 to 14.4 weeks), and a median OS of 23 weeks (range, 16.6 to 29.5 weeks), ${ }^{23}$ which are much similar to our study results. In their study, 34\% of patients were ECOG PS 2, and 72.3\% of the patients had metastatic disease.

Most of the patients tolerated chemotherapy well, with lowgrade adverse events in some patients with poor medical condition. Thirteen of 62 patients (21.0\%) stopped treatment due to the onset of adverse events or worsened general medical condition. Most of the grade 3 adverse events were gastrointestinal 
Table 5. Factors Related to Overall Survival

\begin{tabular}{|c|c|c|c|c|}
\hline & \multicolumn{2}{|c|}{ Univariate analysis } & \multicolumn{2}{|c|}{ Multivariate analysis } \\
\hline & Median OS (95\% CI) & p-value & $\operatorname{HR}(95 \% \mathrm{CI})$ & $\mathrm{p}$-value \\
\hline Sex & & 0.722 & & 0.949 \\
\hline Male & $184(91.4-204.6)$ & & 1 & \\
\hline Female & $158(104.5-211.5)$ & & $1.042(0.546-1.987)$ & \\
\hline Age, yr & & 0.155 & & 0.003 \\
\hline$<65$ & $173(145.1-200.9)$ & & 1 & \\
\hline$\geq 65$ & $117(75.8-158.2)$ & & $2.411(1.297-4.483)$ & \\
\hline Initial tumor stage & & 0.018 & & \\
\hline Recurred tumor & $139(77.0-201.0)$ & & & \\
\hline Stage III & $196(125.7-266.3)$ & & & \\
\hline Stage IV & $122(46.9-197.1)$ & & & \\
\hline Tumor status at failure of first-line treatment & & 0.016 & & 0.004 \\
\hline Locally advanced & $177(116.0-238.0)$ & & 1 & \\
\hline Metastatic & $131(79.2-182.8)$ & & $2.583(1.316-5.069)$ & \\
\hline ECOG performance status & & 0.254 & & \\
\hline 0 & $158(94.4-221.6)$ & & & \\
\hline 1 & $173(147.1-198.9)$ & & & \\
\hline 2 & $104(56.5-151.5)$ & & & \\
\hline Hypertension & & 0.747 & & \\
\hline No & $158(118.0-198.0)$ & & & \\
\hline Yes & $227(64.8-389.2)$ & & & \\
\hline Diabetes & & 0.509 & & \\
\hline No & $129(109.9-148.1)$ & & & \\
\hline Yes & $177(139.0-215.0)$ & & & \\
\hline Initial chemothrapeutic regimen & & 0.105 & & 0.011 \\
\hline Gemcitabine+erlotinib & $187(128.7-245.3)$ & & 1 & \\
\hline Gemcitabine monotherapy & $132(71.6-192.4)$ & & $3.070(1.452-6.490)$ & \\
\hline Gemcitabine+cisplatin & 139 (103.8-174.2) & & $2.309(0.641-8.318)$ & \\
\hline Diagnosis to secondary chemotherapy, day & & 0.006 & & $<0.001$ \\
\hline$<120$ & $117(62.7-171.3)$ & & 1 & \\
\hline$\geq 120$ & $177(136.5-217.5)$ & & $0.265(0.127-0.551)$ & \\
\hline Previous surgical resection & & 0.878 & & \\
\hline No & 158 (119.1-196.9) & & & \\
\hline Yes & $177(108.8-245.2)$ & & & \\
\hline Previous concurrent chemoradiotherapy & & 0.187 & & 0.892 \\
\hline No & $148(100.8-195.2)$ & & 1 & \\
\hline Yes & $187(126.6-247.4)$ & & $0.954(0.448-2.030)$ & \\
\hline
\end{tabular}

OS, overall survival; CI, confidence interval; HR, hazard ratio; ECOG, Eastern Cooperative Oncology Group.

problems (e.g., anorexia, vomiting, diarrhea, and oral mucositis). The frequency of adverse events was slightly more common in our study than in previous studies ${ }^{22,23}$ which may reflect the worse general condition of our study patients at initiation of XELOX combination chemotherapy. There was one treatment related death caused by severe neutropenia and typhlitis; however, the patient was 82 years old at initiation of the treatment, and tumor progression had been documented at the time of death.

In our study, patients who remained stable longer with frontline therapy had significantly longer PFS during second-line XELOX combination therapy. Other factors including sex, age, initial or subsequent tumor stage, previous treatment modality, performance status, or underlying disease were not significantly 
related with PFS. Patients who remained stable longer with frontline therapy also had significantly longer OS. The correlation between longer duration of first-line therapy and longer PFS was already shown in previous studies that have investigated the efficacy of second-line chemotherapy in advanced PDAC patients. ${ }^{25,29}$ This is possibly related to less aggressive tumor biology of such patient.

The limitations of the present study are that the data were collected retrospectively, and no comparative analysis with other second-line therapy was performed. A number of patients (13 of 62, 21.0\%) were not followed up until the first response evaluation. However, we were able to acquire the date of death in all of the patients at the study endpoint, which enables us to reduce the limitations of retrospective study. This study was significant since we included a relatively large number of patients compared with previous studies and many of the included patients were ECOG PS 2, which reflects real practice. We also tried to reveal the significant factors for better prognosis, and some of these factors significantly correlated with better prognosis in multivariate analysis.

In our study, XELOX combination chemotherapy showed acceptable response rate and survival rate, and the adverse events were manageable. We concluded that XELOX combination chemotherapy may a good option as salvage treatment for advanced pancreatic cancer patients whose cancers have progressed on gemcitabine-based chemotherapy.

\section{CONFLICTS OF INTEREST}

No potential conflict of interest relevant to this article was reported.

\section{REFERENCES}

1. Bilimoria KY, Bentrem DJ, Ko CY, et al. Validation of the 6th edition AJCC Pancreatic Cancer Staging System: report from the National Cancer Database. Cancer 2007;110:738-744.

2. Zuckerman DS, Ryan DP. Adjuvant therapy for pancreatic cancer: a review. Cancer 2008;112:243-249.

3. Siegel R, Ma J, Zou Z, Jemal A. Cancer statistics, 2014. CA Cancer J Clin 2014;64:9-29.

4. Yeo CJ, Abrams RA, Grochow LB, et al. Pancreaticoduodenectomy for pancreatic adenocarcinoma: postoperative adjuvant chemoradiation improves survival: a prospective, single-institution experience. Ann Surg 1997;225:621-633.

5. Ducreux M, Cuhna AS, Caramella C, et al. Cancer of the pancreas: ESMO Clinical Practice Guidelines for diagnosis, treatment and follow-up. Ann Oncol 2015;26 Suppl 5:v56-v68.

6. Burris HA 3rd, Moore MJ, Andersen J, et al. Improvements in survival and clinical benefit with gemcitabine as first-line therapy for patients with advanced pancreas cancer: a randomized trial. J Clin Oncol 1997;15:2403-2413.
7. Moore MJ, Goldstein D, Hamm J, et al. Erlotinib plus gemcitabine compared with gemcitabine alone in patients with advanced pancreatic cancer: a phase III trial of the National Cancer Institute of Canada Clinical Trials Group. J Clin Oncol 2007;25:1960-1966.

8. Conroy T, Desseigne F, Ychou M, et al. FOLFIRINOX versus gemcitabine for metastatic pancreatic cancer. N Engl J Med 2011;364: 1817-1825.

9. Colucci G, Labianca R, Di Costanzo F, et al. Randomized phase III trial of gemcitabine plus cisplatin compared with single-agent gemcitabine as first-line treatment of patients with advanced pancreatic cancer: the GIP-1 study. J Clin Oncol 2010;28:1645-1651.

10. Gonçalves A, Gilabert M, François E, et al. BAYPAN study: a double-blind phase III randomized trial comparing gemcitabine plus sorafenib and gemcitabine plus placebo in patients with advanced pancreatic cancer. Ann Oncol 2012;23:2799-2805.

11. Kindler HL, Niedzwiecki D, Hollis D, et al. Gemcitabine plus bevacizumab compared with gemcitabine plus placebo in patients with advanced pancreatic cancer: phase III trial of the Cancer and Leukemia Group B (CALGB 80303). J Clin Oncol 2010;28:3617-3622.

12. Cunningham D, Chau I, Stocken DD, et al. Phase III randomized comparison of gemcitabine versus gemcitabine plus capecitabine in patients with advanced pancreatic cancer. J Clin Oncol 2009;27:5513-5518.

13. Von Hoff DD, Ervin T, Arena FP, et al. Increased survival in pancreatic cancer with nab-paclitaxel plus gemcitabine. N Engl J Med 2013;369:1691-1703.

14. National Comprehensive Cancer Network. NCCN guidelines for treatment of cancer by site: pancreatic adenocarcinoma (version 2.2015). Fort Washington: National Comprehensive Cancer Network, 2015.

15. Tsavaris N, Kosmas C, Skopelitis H, et al. Second-line treatment with oxaliplatin, leucovorin and 5-fluorouracil in gemcitabinepretreated advanced pancreatic cancer: a phase II study. Invest New Drugs 2005;23:369-375.

16. Yoo C, Hwang JY, Kim JE, et al. A randomised phase II study of modified FOLFIRI.3 vs modified FOLFOX as second-line therapy in patients with gemcitabine-refractory advanced pancreatic cancer. Br J Cancer 2009;101:1658-1663.

17. Chung JW, Jang HW, Chung MJ, et al. Folfox4 as a rescue chemotherapy for gemcitabine-refractory pancreatic cancer. Hepatogastroenterology 2013;60:363-367.

18. Oettle H, Riess H, Stieler JM, et al. Second-line oxaliplatin, folinic acid, and fluorouracil versus folinic acid and fluorouracil alone for gemcitabine-refractory pancreatic cancer: outcomes from the CONKO-003 trial. J Clin Oncol 2014;32:2423-2429.

19. Wang-Gillam A, Li CP, Bodoky G, et al. Nanoliposomal irinotecan with fluorouracil and folinic acid in metastatic pancreatic cancer after previous gemcitabine-based therapy (NAPOLI-1): a global, randomised, open-label, phase 3 trial. Lancet 2016;387:545-557.

20. Miwa M, Ura M, Nishida M, et al. Design of a novel oral fluoropyrimidine carbamate, capecitabine, which generates 5-fluorouracil selectively in tumours by enzymes concentrated in human liver 
and cancer tissue. Eur J Cancer 1998;34:1274-1281.

21. Cartwright TH, Cohn A, Varkey JA, et al. Phase II study of oral capecitabine in patients with advanced or metastatic pancreatic cancer. J Clin Oncol 2002;20:160-164.

22. Xiong HQ, Varadhachary GR, Blais JC, Hess KR, Abbruzzese JL, Wolff RA. Phase 2 trial of oxaliplatin plus capecitabine (XELOX) as second-line therapy for patients with advanced pancreatic cancer. Cancer 2008;113:2046-2052.

23. Bayoglu IV, Varol U, Yildiz I, et al. Second-line capecitabine and oxaliplatin combination for gemcitabine-resistant advanced pancreatic cancer. Asian Pac J Cancer Prev 2014;15:7119-7123.

24. Oken MM, Creech RH, Tormey DC, et al. Toxicity and response criteria of the Eastern Cooperative Oncology Group. Am J Clin Oncol 1982;5:649-655.

25. Sinn M, Dälken L, Striefler JK, et al. Second-line treatment in pancreatic cancer patients: who profits? Results from the CONKO
Study Group. Pancreas 2016;45:601-605.

26. Edge SB, Compton CC. The American Joint Committee on Cancer: the 7th edition of the AJCC Cancer Staging Manual and the future of TNM. Ann Surg Oncol 2010;17:1471-1474.

27. Eisenhauer EA, Therasse P, Bogaerts J, et al. New response evaluation criteria in solid tumours: revised RECIST guideline (version 1.1). Eur J Cancer 2009;45:228-247.

28. Trotti A, Colevas AD, Setser A, et al. CTCAE v3.0: development of a comprehensive grading system for the adverse effects of cancer treatment. Semin Radiat Oncol 2003;13:176-181.

29. Herrmann C, Abel U, Stremmel W, Jaeger D, Herrmann T. Short time to progression under first-line chemotherapy is a negative prognostic factor for time to progression and residual survival under second-line chemotherapy in advanced pancreatic cancer. Oncology 2007;73:335-339. 